\title{
Anti-inflammatory Property of the Essential Oil From Cinnamomum Camphora (Linn.) Presl Leaves and the Evaluation of Its Underlying Mechanism by Using Metabolomics Analysis
}

Jiali Chen

School of Pharmaceutical Sciences, Sun Yat-Sen University

Cailin Tang

School of Pharmaceutical Sciences, Sun Yat-Sen University

Yang Zhou

School of Pharmaceutical Sciences, Sun Yat-Sen University

Rongfei Zhang

School of Pharmaceutical Sciences, Sun Yat-Sen University

Shaoxia Ye

School of Pharmaceutical Sciences, Sun Yat-Sen University

Zhimin Zhao

School of Pharmaceutical Sciences, Sun Yat-Sen University

Ligen Lin

State Key Laboratory of Quality Research in Chinese Medicine: University of Macau Institute of Chinese Medical Science

Depo Yang ( $\square$ lssydp@mail.sysu.edu.cn )

School of Pharmaceutical Sciences, Sun Yat-Sen University, Guangzhou 510006 Guangdong Province, People Republic of China

\section{Research}

Keywords: Essential oil, Cinnamomum camphora (Linn.) Presl, Anti-inflammatory, BV2 microglial cells, Metabolomics, Glycolysis

Posted Date: September 17th, 2020

DOI: https://doi.org/10.21203/rs.3.rs-76716/v1

License: (9) (1) This work is licensed under a Creative Commons Attribution 4.0 International License. Read Full License 


\section{Abstract}

Background: Cinnamomum camphora (Linn.) Presl has been widely used in traditional Chinese medicine for a variety of purposes. Our previous study indicated the antibacterial mechanism of the essential oil (EO) from C. camphora; however, the anti-inflammatory activity of EO and its underlying mechanism have not been clearly demonstrated. The present study aims to evaluate the anti-inflammatory principle and mechanism of EO.

Methods: The anti-inflammatory activity of EO was evaluated in lipopolysaccharide (LPS)-induced BV2 microglial cells. Nitric oxide (NO) production was measured by NO assay kit. The mRNA expression levels of inducible NO synthase (iNOS), interleukin-6 (IL-6), IL-18 and IL-1 $\beta$ were examined by real time-PCR (RTPCR). The secretion of pro-inflammatory cytokines in cell supernatants, including IL-6, IL-18 and IL-1 $\beta$, were assessed by ELISA kits. Furthermore, the metabolic profile of BV2 microglial cells treated with or without EO was explored by GC-MS-based metabolomics analysis. Phosphofructokinase (PFK) and pyruvate kinase (PK) activities were detected by commercial kits.

Results: EO significantly decreased the release of NO and the mRNA expression of iNOS in LPS-induced BV2 microglial cells. EO also attenuated LPS-induced increase in the mRNA expression and secretion of inflammatory cytokines including IL-6, IL-18 and IL-1 $\beta$. 39 metabolites were identified with significantly different contents, including 21 upregulated and 18 downregulated ones, in the metabolomics analysis. Five pathways were enriched by shared differential metabolites. Additionally, compared with the control group, the glucose level was decreased, while the lactate level was increased, in the culture supernatant of LPS-induced BV2 microglia cells, which were reversed by EO treatment. Besides, compared to the LPStreated group, the activities for PK and PFK in EO group were decreased by $17.59 \%$ and $18.23 \%$, respectively.

Conclusions: The EO from C. camphora acts as an anti-inflammatory agent, which might be mediated through attenuating the glycolysis capacity of microglial cells.

\section{Introduction}

Cinnamomum camphora (Linn.) Presl, commonly known as camphor tree or camphor laurel, is widely cultivated in Southern China as an ornamental plant and a material for wood furniture ${ }^{1}$. In traditional Chinese medicine, the essential oil from this plant (EO) has been widely used to treat inflammation, rheumatic conditions and muscular strains ${ }^{2}$. A growing number of pharmacological researches have shown that $C$. camphora exhibits a wide range of biological activities such as anti-inflammation, antibacteria, antioxidation, anti-fungi, and repellent properties ${ }^{3-11}$. In our previous study ${ }^{12}, 48$ constituents from EO were identified; and linalool was the major compound (26.6\%), followed by eucalyptol (16.8\%), aterpineol (8.7\%), isoborneol (8.1\%), $\beta$-phellandrene (5.1\%), and camphor $(5.0 \%)$. 
Inflammation, a complex series of interactions among cells and soluble factors, causes persistent tissue damage by leukocytes, lymphocytes or collagen ${ }^{13}$. Dysregulated inflammation, such as long-term chronic inflammation, has been considered to play key roles in the onset and development of metabolic disorders, neurodegeneration, and cardiovascular diseases ${ }^{13,14}$. It is of great significance to attenuate the increases of various inflammatory mediators, including tumor necrosis factor- $a$ (TNF-a), interleukin (IL)-6, IL-1 $\beta$, and nitric oxide (NO) ${ }^{15-17}$. In addition, inflammatory response is usually associated with metabolic changes ${ }^{18}$. Metabolomics is considered to be a powerful platform to explore the potential mechanisms of drugs at the biochemical level. A recent study investigated the anti-neuroinflammatory potential of Clinacanthus nutans leaf extracts towards lipopolysaccharide (LPS)-induced BV2 microglial cells by using metabolomics approach ${ }^{19}$. The anti-inflammatory activity of EO and its potential mechanisms remain largely unelucidated.

In recent years, increasing evidence indicates that glycolysis plays an important role in the development of inflammation ${ }^{20,21}$. Glycolysis, a ubiquitous metabolic pathway in mammals, is regulated by multiple glycolytic enzymes, including phosphofructokinase (PFK) and pyruvate kinase (PK) ${ }^{22,23}$. Activation of the glycolytic pathway provides essential biomacromolecules, including amino acids and fatty acids, to synthesize inflammatory mediators in cells. LPS-induced cells undergo changes in glycolytic metabolism

24 . The increased level of lactate is also related to glycolytic metabolism ${ }^{25,26}$. Furthermore, lactate has been reported to enhance LPS-stimulated proinflammatory responses ${ }^{27}$. Therefore, the anti-inflammatory activity of molecules might be related to their effect on glycolytic pathway. Previous study showed that lidocaine inhibits the secretion of inflammatory cytokines in LPS-induced peritoneal macrophages by inhibiting the glycolytic pathway ${ }^{28}$.

In order to assess the anti-inflammatory activity of EO and explore the underlying mechanisms, this study was designed to detect NO production, cytokines secretion, and mRNA expression of inducible NO synthase (iNOS), IL-6, IL-18, and IL-1 $\beta$ in LPS-induced BV2 microglial cells. Furthermore, GC-MS-based metabolomics analysis and the glycolytic enzyme activity were investigated. Herein, we desire to provide better insight into the anti-inflammatory potential of EO.

\section{Materials And Methods}

\section{Reagents}

3-(4,5-dimethylthiazol-2-yl)-2,5-diphenyltetrazolium bromide (MTT), dimethyl sulfoxide (DMSO), trypsin, and LPS were acquired from Sigma-Aldrich (St. Louis, MO, USA). Penicillin and streptomycin were purchased from Hyclone (Logan, UT, USA). Dulbecco's modified Eagle's medium (DMEM), fetal bovine serum (FBS), and Trizol RNA extraction reagent were obtained from Invitrogen (Grand Island, NY, USA). BCA protein kit and NO assay kit were obtained from Beyotime Institute of Biotechnology (Jiangsu, China). The ELISA kits for IL-18, IL-1 $\beta$ and IL-6 were purchased from Boster Biological Technology (Wuhan, China). Hieff ${ }^{\text {Tw }}$ qPCR SYBR ${ }^{\circledR}$ Green Master Mix (Yisheng, Shanghai, China), glucose and lactate 
assay kits were acquired from Nanjing Jiancheng Bioengineering Institute (Nanjing, China). The PFK and PK activity assay kits were purchased from Beijing Solarbio Science \& Technology Co., Ltd (Beijing, China).

\section{Sample collection and extraction}

Leaves of C. camphora were collected on March 2019 from adult trees located in the campus of Sun YatSen University, and identified by Professor Depo Yang, School of Pharmaceutical Sciences, Sun Yat-Sen University. A voucher specimen for C. camphora (NO. A20190301) has been deposited at School of Pharmaceutical Sciences, Sun Yat-Sen University. The EO was extracted by hydrodistillation in a Clevenger-type apparatus for $2 \mathrm{~h}$, and then dehydrated by filtration with anhydrous sodium sulfate.

\section{Cell culture}

The BV2 microglial cells were obtained from the Cell Bank of Shanghai Institute of Biochemistry and Cell Biology (Chinese Academy of Sciences, Shanghai, China). Cells were cultured in DMEM with $10 \%$ FBS, $100 \mathrm{U} / \mathrm{mL}$ penicillin, and $100 \mathrm{~g} / \mathrm{mL}$ streptomycin in a humidified incubator (Thermo Fisher, San Diego, CA, USA) containing $5 \% \mathrm{CO}_{2}$ at $37{ }^{\circ} \mathrm{C}$. A TPVG solution ( $0.2 \%$ trypsin, $0.05 \%$ glucose and $0.2 \%$ EDTA in phosphate buffer saline solution) was used for cell dissociation.

\section{Determination of cell viability}

Cell viability was measured by MTT assay as described in previously reported protocol ${ }^{29}$.

\section{Measurement of NO production}

BV2 microglial cells $\left(5.5 \times 10^{4}\right.$ cells/well) were incubated in a 96 -well plate for $24 \mathrm{~h}$ and then treated with EO at different concentrations in the absence or presence of LPS $(25 \mathrm{ng} / \mathrm{mL})$. The cells treated with LPS alone were set as controls, and those treated with neither EO nor LPS were set as blank controls. After $24 \mathrm{~h}$, NO production was determined by measuring the amount of nitrite in cell culture supernatants using the nitric oxide assay kit.

\section{Measurement of IL-18, IL-1 $\beta$ and IL-6 production}

BV2 microglial cells $\left(5 \times 10^{4}\right.$ cells/well) were seeded in a 12-well plate and incubated for $24 \mathrm{~h}$. After treated with EO at different concentrations $(100,150,200 \mu \mathrm{g} / \mathrm{mL})$ and stimulated with LPS $(25 \mathrm{ng} / \mathrm{mL})$ for $24 \mathrm{~h}$, the release of IL-18, IL-1 $\beta$ and IL- 6 in the culture supernatants was quantified by using the corresponding ELISA kits according to the manufacturers' protocols.

\section{Total RNA extraction and RT-PCR}

BV2 microglial cells $\left(1 \times 10^{6}\right.$ cells/well) were incubated into 6 -well plates for $24 \mathrm{~h}$. The cells were treated with different concentrations of EO in the presence or absence of LPS $(25 \mathrm{ng} / \mathrm{mL})$ for $24 \mathrm{~h}$. The RNA samples were isolated with Trizol reagent, and reversely transcribed into complementary DNA using HiScript II Q RT SuperMix for qPCR. Quantitative RT-PCR was performed using the Hieff ${ }^{\mathrm{Tm}}$ qPCR SYBR ${ }^{\circ}$ Green Master Mix according to the manufacturer's protocol and detected by the LightCycler 96 Real-Time 
PCR System. All experiments were performed in triplicate. Relative mRNA levels were calculated using $2^{-\triangle \Delta C t}$ method. GAPDH was used as a housekeeping gene. The primer sequences were shown in Table 1 .

Table 1

Primer sequences.

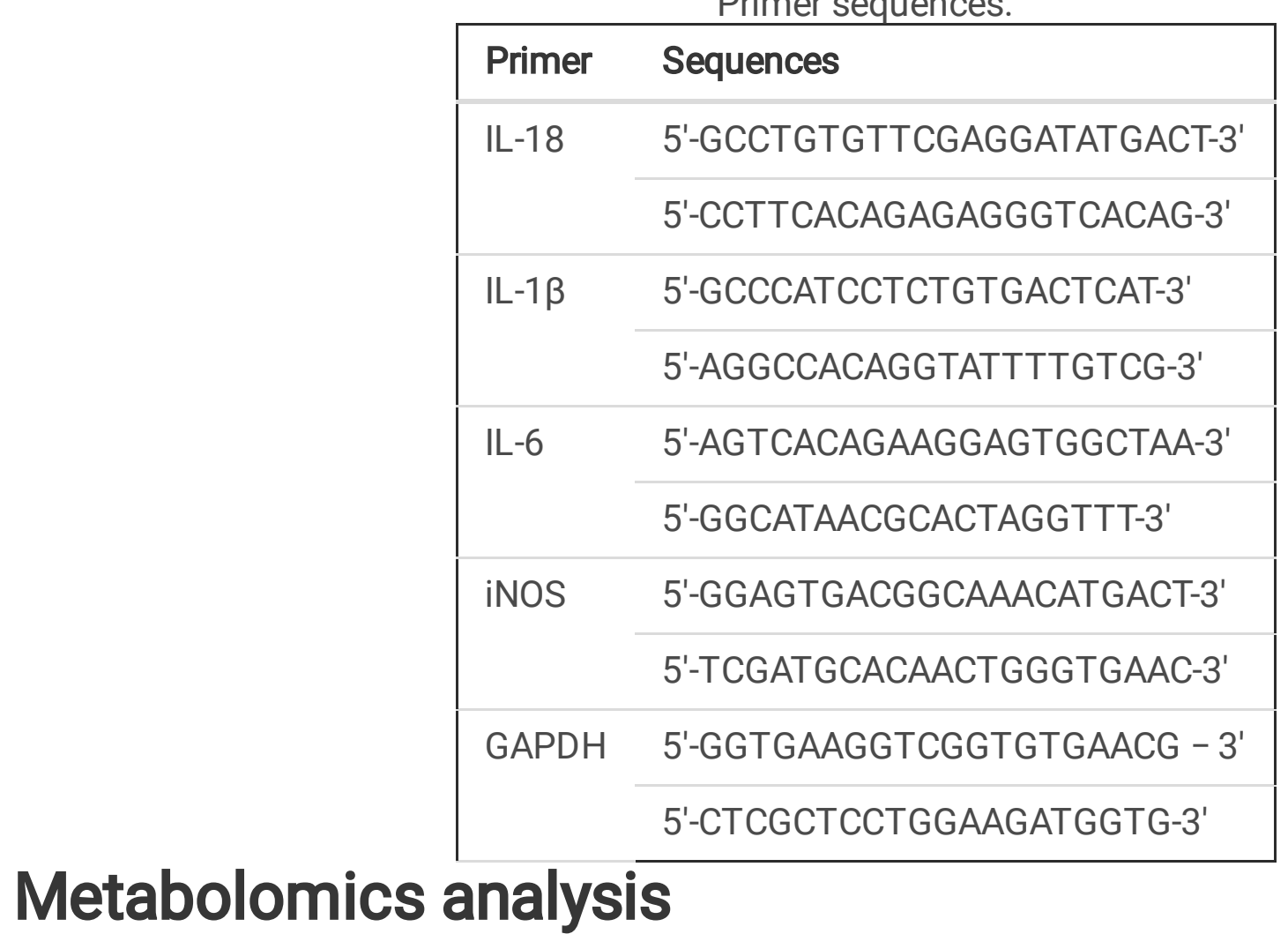

BV2 microglial cells were collected by centrifugation at 1,000 rpm for $3 \mathrm{~min}$, washed with PBS for three times, and resuspended in methanol. The cells were homogenized by ultrasonic, followed by centrifugation at $12,000 \mathrm{rpm}$ at $4{ }^{\circ} \mathrm{C}$ for $10 \mathrm{~min}$. The supernatant of each sample was collected, and 10 $\mu \mathrm{L}$ ribitol $(0.1 \mathrm{mg} / \mathrm{mL})$ was added as the internal standard for analysis. Then the supernatants were concentrated in a rotary vacuum centrifuge device (LABCONCO). The dried extracts were used for GC-MS analysis, as described in previous study ${ }^{12}$. Each sample contained six biological and two technical replicates.

\section{Measurement of lactate and glucose levels}

BV2 microglial cells were treated under the same conditions as those of RNA extraction. Lactate and glucose levels in the culture supernatants were estimated by using the lactate and glucose assay kits, respectively.

\section{Measurement of enzyme activity}

Cells were collected by centrifugation at 1,000 rpm for $3 \mathrm{~min}$ and then washed with PBS for three times. The PFK and PK activities were analyzed spectrophotometrically by using commercially available kits according to manufacturer's instructions. Inhibition rate $\%=[($ sample - control $) /$ control $] * 100 \%$. 


\section{Statistical analysis}

All data were expressed as mean \pm SD with the statistical method of one-way analysis of variance (ANOVA) and Student's t-test using the SPSS 19.0 software. A $P$ value less than 0.05 was considered as a statistically significant difference. Metabolomics data was analyzed based on the technique described in our previous study ${ }^{12}$.

\section{Results}

\section{Cytotoxic effect of EO on BV2 microglial cells}

To exclude the cytotoxicity, the BV2 microglial cells were treated with EO at concentrations ranging of $7.81-250 \mu \mathrm{g} / \mathrm{mL}$ for $48 \mathrm{~h}$. The viability of BV2 microglial cells was measured by the MTT assay. The results showed that EO did not obviously affect cell viability even at a concentration up to $250 \mu \mathrm{g} / \mathrm{mL}$ Thus, the doses of $7.81-250 \mu \mathrm{g} / \mathrm{mL}$ of EO were selected for further experiments.

\section{Effects of EO on the production of $\mathrm{NO}$ and the expression of iNOS}

In order to investigate the anti-inflammatory effect of EO, NO production was evaluated in LPS-induced BV2 microglial cells. As shown in Fig. 1A, the NO production in LPS exposed cells was significantly increased, in compared with the control group, whereas EO treatment dose-dependently decreased NO production in BV2 microglial cells. Subsequently, RT-PCR results showed that EO suppressed the LPSinduced increase of iNOS expression in a dose-dependent manner (Fig. 1B). These results indicated that EO inhibited NO production through downregulating iNOS mRNA level in LPS-induced BV2 microglial cells.

\section{Effects of EO on the expression and secretion of IL-6, IL-18 and IL-1 $\beta$}

To confirm the anti-inflammatory effect of EO, the levels of inflammatory mediators, including IL-6, IL-18 and IL-1 $\beta$, were evaluated on LPS-stimulated BV2 microglial cells. Compared with the control group, LPS increased IL-6, IL-18 and IL-1 $\beta$ transcription, which were decreased after treated with EO in dosedependent manners (Fig. 1C-E). The ELISA results indicated that the secretion of IL-6, IL-18 and IL-1 $\beta$ in cell supernatants were remarkably increased in LPS group compared with the control; and EO treatment alleviated the elevated levels of IL-6, IL-18 and IL-1 $\beta$ (Fig. 1F-H).

\section{Metabolomics analysis}

Six biological and two technical replicates yielded 36 data in this study. There were 60 metabolites for subsequent analysis after data processing. KEGG was used to retrieve the biological categories of the identified metabolites. The abundance of 39 metabolites in the EO group was different in comparison of 
the model group (Fig. 2A). The Z-value was calculated for a comparative study based on the model group, showing the variations of these metabolites. The Z-score plot displayed that it spanned from 7.74 to 7.37 in the EO group (Fig. 2B). Specifically, there were 18 metabolites decreased and 21 metabolites increased in the EO group, when compared with the model group.

To investigate the metabolic differences among the control group, model group and EO group, orthogonal partial least-squares discriminant analysis (OPLS-DA) was applied for the recognition of the sample patterns, followed by ranking the altered metabolites in loading. The three groups were separated obviously. (Fig. 3A). Discriminating variables were present with S-plot (Fig. 3B) while cut-off values were set as greater or equal to the 0.05 and 0.5 for the absolute value of covariance $p$ and correlation $p$ (corr), respectively. Alanine, hexadecanoic acid, aspartic acid, valine, and myo-inositol showed the largest correlations and covariances in the predictive component between the model group and the EO group (Fig. 3C).

Five impacted pathways were enriched by shared differential metabolites, including alanine, aspartate and glutamate metabolism, citrate cycle (TCA cycle), galactose metabolism, fatty acid biosynthesis, and pantothenate and CoA biosynthesis, between the model and EO groups (Fig. 4A). The first four pathways were dramatically impacted $(P<0.05)$, where amino acid metabolism was the most significant one, followed by glucose metabolism. As shown in Fig. 4B, most of metabolites enriched in the five pathways were decreased. The alanine aspartate and glutamate metabolism pathway was mostly impacted among the altered metabolic pathways under EO treatment $(P<0.05)$. These results indicated that antiinflammatory activity of EO might be through interfering with amino acid and glucose metabolism.

\section{Analysis of lactate and glucose levels}

To evaluate whether glycolytic activity was suppressed by EO treatment, lactate production and glucose uptake were detected in BV2 microglial cells. Compared with the control group, the glucose level was decreased, while the lactate level was increased, in the culture supernatant of LPS-induced BV2 microglia cells, indicating that LPS treatment augmented the glycolytic capacity; however, the glucose level was increased and the lactate level was decreased after EO treatment, which indicated that EO suppressed glycolytic capacity induced by LPS (Fig. 5).

\section{Enzyme activity analysis}

To further demonstrate the effect of EO on glycolysis, the activities of PK and PFK, key enzymes associated with glycolysis, were measured. As shown in Fig. 6, compared to the control group, the activities for PK and PFK increased by $3.96 \%, 95.74 \%$ and $24.88 \%$ after treatment with LPS, respectively, whereas, the activities for PK and PFK decreased by $17.59 \%$ and $18.23 \%$ after EO treatment. It suggested that EO could disturb the LPS-induced activation of glycolysis.

\section{Discussion}


Inflammation, an important protective response of organism, aims to eliminate or scavenge harmful stimuli, including damaged cells and pathogens, so as to restore normal tissue structure and function. A wild inflammation causes harmful effects to tissues and microcirculatory systems, and leads to severe complications ${ }^{13}$. Therefore, alleviation of inflammatory responses is the key for tissues to recover from different stimulating factors ${ }^{30}$. NO plays an important role in the immune system and the maintenance of body quiescence, but excessive NO causes a variety of inflammatory related diseases. In addition, the upregulation of inflammatory factors persisting at an inflammatory site is important, such as IL-1 $\beta$, IL-18 and IL-6. Overproduction of these mediators recruits downstream effector cells (such as neutrophils), and directs the natural evolution of the inflammatory response ${ }^{31}$. Therefore, it is of great significance to explore the mechanisms of inflammation by detecting and observing the changes of these inflammatory factors ${ }^{32}$. The model of LPS-induced NO production in BV2 microglial cells is widely employed in screening anti-inflammatory agents for neuroinflammation and related neurodegenerative diseases ${ }^{33}$. Kim et al. found that ginsenosides exert anti-inflammatory activities by suppressing the inflammatory enzymes, such as iNOS and COX-2, as well as the production of proinflammatory cytokinases such as TNF-a, IL-1 $\beta$, and IL-6, in LPS-stimulated macrophages and microglial cells ${ }^{30}$. Currently, EO was found to inhibit LPS-induced NO production via suppression of iNOS expression at the mRNA level. In addition, the production of pro-inflammatory cytokines (IL-16, IL-18, and IL-1 $\beta$ ) was significantly reduced by EO in BV2 microglial cells. These results showed that EO notably inhibits the expression of pro-inflammatory mediators, suggesting that it could inhibit the occurrence of inflammatory responses or reduce the aggravation of inflammatory mediators.

Recent findings suggested that inflammation is closely related to metabolic disorders, thus the metabolites of biological organisms can be used as important indicators to reflect the inhibition or activation of related metabolic pathways under inflammation ${ }^{34}$. The significant disparation of metabolites can be witnessed by metabolomics profiling analysis ${ }^{21}$. Previous investigations have shown that LPS activates BV2 microglial cells resulting in altered metabolism. Therefore, this study applied metabolomics to elucidate the anti-inflammatory mechanism of EO in LPS-induced BV2 microglial cells. A previous study showed the changes in the metabolite levels were related to the anti-inflammatory effects of volatile oils from Angelica sinensis (VOAS), after the intervention of VOAS in acute inflammation displayed a trend of restoring the level of biomarkers to normal ${ }^{35}$. Similarly, the relative concentrations of metabolites between the model group and the EO group further demonstrated that physiological metabolism of LPS-induced BV2 microglial cells was restored after EO treatment. According to the metabolomics analysis, most were amino acids among the 39 significantly different metabolites, which play essential roles in an organism. Further enrichment analysis and pathway analysis pointed mainly to the involvement of amino acid, glucose metabolism and fatty acid metabolism. Based on metabolic pathway analysis, TCA cycle plays the most important role in the amino acid metabolism, and the major metabolic part of galactose metabolism is glycolysis ${ }^{35}$.

Recent studies have shown that pro-inflammatory responses of microglia are driven by glycolysis, which is associated with high level of glucose uptake, revealing the crucial function of glycolysis in 
inflammation responses ${ }^{16}$. Lactate is an important product of glycolysis, and high lactate level is associated with a number of cellular events that lead to inflammation ${ }^{27}$. Therefore, the contents of glucose and lactate in the cell supernatant can reflect the glycolytic capacity of BV2 microglial cells. The experimental results showed that EO inhibits LPS-induced increase of glucose consumption and lactate production, suggesting that EO might inhibit the glycolytic ability in LPS-induced BV2 microglia cells. Growing evidence indicates that glycolysis is closely linked with several key enzymes, which are crucial for the regulation of glycolysis, including PFK and $\mathrm{PK}^{18}$. Wang et al. demonstrated that the deacetylase Sirtuin 5 plays a role in regulating inflammatory response by regulating the PK activity of PKM2; besides, withaferin A, a bioactive compound derived from Withania somnifera, considerably improved hepatic inflammation by regulating glycolysis-related enzyme genes in the liver of obese mice ${ }^{36}$. Similarly, our results verified that cells treated with EO suffered a decrease in the activities of PK and PFK and disrupted glycolysis. Glycolysis converts glucose into pyruvate, which is subsequently converted into lactate, and secreted, or gets into TCA cycle, which pointed out the importance of glycolysis. Therefore, further study about the factors affecting glycolysis can be the direction of our research.

\section{Conclusions}

In summary, a metabolomics approach was adopted to investigate the anti-inflammatory mechanisms of EO. Our studies showed that EO exerts anti-inflammatory effects by inhibiting iNOS gene expression and NO production, as well as the expression and secretion of inflammatory cytokines (IL-6, IL-18 and IL-1 $\beta$ ) in LPS-treated BV2 microglial cells. We demonstrated that LPS promotes significant metabolic changes, resulting in increasing glycolysis. Whereas, EO suppresses the increase of glucose consumption and lactate production in LPS-stimulated BV2 microglial cells, which was related to the decreasing activities of key enzymes in glycolysis. Therefore, these results elucidated that EO acts as an anti-inflammatory agent via attenuating the LPS-induced glycolysis capacity. Furthermore, these data elucidated a molecular pathway that directly links glycolysis to the anti-inflammatory program of BV2 microglial cells, suggesting a potential role for metabolic therapies in treating inflammation.

\section{Abbreviations}

EO, essential oil; GC-MS, Gas chromatography-mass spectrometry; IL-18, Interleukin-18; IL-1ß, Interleukin1ß; IL-6, Interleukin-6; KEGG, Kyoto Encyclopedia of Genes and Genomes Database; MTT, Thiazolyl blue tetrazolium bromide; NO, Nitric oxide; OPLS-DA, Orthogonal projections to latent structures discriminant analysis; PBS, phosphate buffer saline; PCA, Principal component analysis; PFK, phosphofructokinase; PK, pyruvate kinase; rpm, revolutions per minute; PLS-DA, Partial least squares-discriminant analysis; RTPCR, real-time PCR; TCA, tricarboxylic acid;

\section{Declarations}

Acknowledgments 
Not applicable.

\section{Authors' contributions}

Jiali Chen designed the research, performed the experiment and wrote the manuscript. Cailin Tang, Yang Zhou, Rongfei Zhang and Shaoxia Ye revised drafts of the manuscript. Depo Yang, Zhimin Zhao and Ligen Lin supervised the work and reviewed the manuscript. All authors read and approved the final manuscript.

\section{Funding}

This work was financially supported by the National Key R\&D Program of China (NO. 2017YFC1701100) and Guangzhou Science and Technology Project (NO. 201904010203).

\section{Data availability statement}

The datasets used in this study are available from the corresponding author upon reasonable request.

\section{Ethics approval and consent to participate}

Not applicable.

\section{Consent for publication}

Not applicable.

\section{Competing interests}

The authors declare that they have no competing financial interests.

\section{Author details}

${ }^{1}$ School of Pharmaceutical Sciences, Sun Yat-Sen University, Guangzhou 510006 Guangdong Province, People Republic of China.

2 State Key Laboratory of Quality Research in Chinese Medicine, Institute of Chinese Medical Sciences, University of Macau, Avenida da Universidade, Taipa, Macau 999078, China.

\section{References}

1. Chen, S.; Zheng, T.; Ye, C.; Huannixi, W.; Yakefu, Z.; Meng, Y.; Peng, X.; Tian, Z.; Wang, J.; Ma, Y.; Yang, Y.; Ma, Z.; Zuo, Z., Algicidal properties of extracts from Cinnamomum camphora fresh leaves and their main compounds. Ecotoxicol Environ Saf 2018, 163, 594-603.

2. Guo, S.; Geng, Z.; Zhang, W.; Liang, J.; Wang, C.; Deng, Z.; Du, S., The chemical composition of essential oils from Cinnamomum camphora and their insecticidal activity against the stored product 
pests. Int J Mol Sci 2016, 17, (11), 1836.

3. Yeh, R. Y.; Shiu, Y. L.; Shei, S. C.; Cheng, S. C.; Huang, S. Y.; Lin, J. C.; Liu, C. H., Evaluation of the antibacterial activity of leaf and twig extracts of stout camphor tree, Cinnamomum kanehirae, and the effects on immunity and disease resistance of white shrimp, Litopenaeus vannamei. Fish Shellfish Immunol 2009, 27, (1), 26-32.

4. Kang, N. J.; Han, S. C.; Yoon, S. H.; Sim, J. Y.; Maeng, Y. H.; Kang, H. K.; Yoo, E. S., Cinnamomum camphora leaves alleviate allergic skin inflammatory responses in vitro and in vivo. Toxicol Res 2019, 35, (3), 279-285.

5. Jiang, H.; Wang, J.; Song, L.; Cao, X.; Yao, X.; Tang, F.; Yue, Y., GCxGC-TOFMS Analysis of Essential Oils Composition from Leaves, Twigs and Seeds of Cinnamomum camphora L. Presl and their Insecticidal and Repellent Activities. Molecules 2016, 21, (4), 423.

6. Pragadheesh, V. S.; Saroj, A.; Yadav, A.; Chanotiya, C. S.; Alam, M.; Samad, A., Chemical characterization and antifungal activity of Cinnamomum camphora essential oil. Ind Crops Prod 2013, 49, 628-633.

7. Singh, R.; Jawaid, T., Cinnamomum camphora (Kapur): Review. Pharmacognosy Journal 2012, 4, (28), 1-5.

8. Lee, H. J.; Hyun, E. A.; Yoon, W. J.; Kim, B. H.; Rhee, M. H.; Kang, H. K.; Cho, J. Y.; Yoo, E. S., In vitro antiinflammatory and anti-oxidative effects of Cinnamomum camphora extracts. J Ethnopharmacol 2006, 103, (2), 208-16.

9. Li, Y. R.; Fu, C. S.; Yang, W. J.; Wang, X. L.; Feng, D.; Wang, X. N.; Ren, D. M.; Lou, H. X.; Shen, T., Investigation of constituents from Cinnamomum camphora (L.) J. Presl and evaluation of their antiinflammatory properties in lipopolysaccharide-stimulated RAW 264.7 macrophages. J Ethnopharmacol 2018, 221, 37-47.

10. Shahlari, M.; Hamidpour, M.; Hamidpour, S.; Hamidpour, R., Camphor (Cinnamomum camphora), a traditional remedy with the history of treating several diseases. J Case Rep Clin Images 2013, 4, (2).

11. Shen, Y.-C.; Chou, C.-J.; Wang, Y.-H.; Chen, C.-F.; Chou, Y.-C.; Lu, M.-K., Anti-inflammatory activity of the extracts from mycelia of Antrodia camphoratacultured with water-soluble fractions from five different Cinnamomums pecies. FEMS Microbiol Lett 2004, 231, (1), 137-143.

12. Chen, J.; Tang, C.; Zhang, R.; Ye, S.; Zhao, Z.; Huang, Y.; Xu, X.; Lan, W.; Yang, D., Metabolomics analysis to evaluate the antibacterial activity of the essential oil from the leaves of Cinnamomum camphora (Linn.) Presl. J Ethnopharmacol 2020, 253, 112652.

13. Nathan, C., Points of control in inflammation. Nature 2002, 420, (6917), 846-52.

14. Ashley, N. T.; Weil, Z. M.; Nelson, R. J., Inflammation: Mechanisms, Costs, and Natural Variation. Annu Rev Ecol Evol Syst 2012, 43, (1), 385-406.

15. Das, A.; Kim, S. H.; Arifuzzaman, S.; Yoon, T.; Chai, J. C.; Lee, Y. S.; Park, K. S.; Jung, K. H.; Chai, Y. G., Transcriptome sequencing reveals that LPS-triggered transcriptional responses in established microglia BV2 cell lines are poorly representative of primary microglia. J Neuroinflammation 2016, $13,(1), 182$. 
16. Wang, L.; Pavlou, S.; Du, X.; Bhuckory, M.; Xu, H.; Chen, M., Glucose transporter 1 critically controls microglial activation through facilitating glycolysis. Mol Neurodegener 2019, 14, (1), 2.

17. Serhan, C. N.; Chiang, N.; Van Dyke, T. E., Resolving inflammation: dual anti-inflammatory and proresolution lipid mediators. Nat Rev Immunol 2008, 8, (5), 349-61.

18. O'Neill, L. A.; Kishton, R. J.; Rathmell, J., A guide to immunometabolism for immunologists. Nat Rev Immunol 2016, 16, (9), 553-65.

19. Ahmad Azam, A.; Ismail, I. S.; Vidyadaran, S.; Abas, F.; Shaari, K., 1H NMR-Based Metabolomics of Clinacanthus nutans Leaves Extracts in Correlation with Their Anti-neuroinflammation Towards LPSInduced BV2 Cells. Rec Nat Prod 2020, 14, (4), 231-247.

20. Orihuela, R.; McPherson, C. A.; Harry, G. J., Microglial M1/M2 polarization and metabolic states. Br J Pharmacol 2016, 173, (4), 649-65.

21. Wang, T.; Liu, H.; Lian, G.; Zhang, S. Y.; Wang, X.; Jiang, C., HIF1alpha-Induced Glycolysis Metabolism Is Essential to the Activation of Inflammatory Macrophages. Mediators Inflamm 2017, 2017, 9029327.

22. Wu, Z.; Wu, J.; Zhao, Q.; Fu, S.; Jin, J., Emerging roles of aerobic glycolysis in breast cancer. Clin Transl Oncol 2020, 22, (5), 631-646.

23. Li, X. B.; Gu, J. D.; Zhou, Q. H., Review of aerobic glycolysis and its key enzymes - new targets for lung cancer therapy. Thorac Cancer 2015, 6, (1), 17-24.

24. Yu, Q.; Wang, Y.; Dong, L.; He, Y.; Liu, R.; Yang, Q.; Cao, Y.; Wang, Y.; Jia, A.; Bi, Y.; Liu, G., Regulations of Glycolytic Activities on Macrophages Functions in Tumor and Infectious Inflammation. Front Cell Infect Microbiol 2020, 10, 287.

25. Jiao, L.; Wang, S.; Zheng, Y.; Wang, N.; Yang, B.; Wang, D.; Yang, D.; Mei, W.; Zhao, Z.; Wang, Z., Betulinic acid suppresses breast cancer aerobic glycolysis via caveolin-1/NF-kappaB/c-Myc pathway. Biochem Pharmacol 2019, 161, 149-162.

26. Brooks, G. A., Lactate as a fulcrum of metabolism. Redox Biol 2020, 35, 101454.

27. Nareika, A.; He, L.; Game, B. A.; Slate, E. H.; Sanders, J. J.; London, S. D.; Lopes-Virella, M. F.; Huang, Y., Sodium lactate increases LPS-stimulated MMP and cytokine expression in U937 histiocytes by enhancing AP-1 and NF-kappaB transcriptional activities. Am J Physiol Endocrinol Metab 2005, 289, (4), E534-42.

28. Lin, S.; Jin, P.; Shao, C.; Lu, W.; Xiang, Q.; Jiang, Z.; Zhang, Y.; Bian, J., Lidocaine attenuates lipopolysaccharide-induced inflammatory responses and protects against endotoxemia in mice by suppressing HIF1alpha-induced glycolysis. Int Immunopharmacol 2020, 80, 106150.

29. Ko, W.; Sohn, J. H.; Jang, J. H.; Ahn, J. S.; Kang, D. G.; Lee, H. S.; Kim, J. S.; Kim, Y. C.; Oh, H., Inhibitory effects of alternaramide on inflammatory mediator expression through TLR4-MyD88-mediated inhibition of NF-small ka, CyrillicB and MAPK pathway signaling in lipopolysaccharide-stimulated RAW264.7 and BV2 cells. Chem Biol Interact 2016, 244, 16-26.

30. Kim, J. H.; Yi, Y. S.; Kim, M. Y.; Cho, J. Y., Role of ginsenosides, the main active components of Panax ginseng, in inflammatory responses and diseases. J Ginseng Res 2017, 41, (4), 435-443. 
31. Coussens, L. M.; Werb, Z., Inflammation and cancer. Nature 2002, 420, (6917), 860-7.

32. Ning, Q.; Liu, Z.; Wang, X.; Zhang, R.; Zhang, J.; Yang, M.; Sun, H.; Han, F.; Zhao, W.; Zhang, X., Neurodegenerative changes and neuroapoptosis induced by systemic lipopolysaccharide administration are reversed by dexmedetomidine treatment in mice. Neurol Res 2017, 39, (4), 357366.

33. Henn A, L. S., Hedtjärn M, Schrattenholz A, Pörzgen P, Leist M, The Suitability of BV2 Cells as Alternative Model System for Primary Microglia Cultures or for Animal Experiments Examining Brain Inflammatio. ALTEX 2009, 26, (2), 83-94.

34. Gong, L.; Yu, L.; Gong, X.; Wang, C.; Hu, N.; Dai, X.; Peng, C.; Li, Y., Exploration of anti-inflammatory mechanism of forsythiaside $\mathrm{A}$ and forsythiaside $\mathrm{B}$ in $\mathrm{CuSO}_{4}$-induced inflammation in zebrafish by metabolomic and proteomic analyses. J Neuroinflammation 2020, 17, (1), 173.

35. Zhang, W. Q.; Hua, Y. L.; Zhang, M.; Ji, P.; Li, J. X.; Zhang, L.; Li, P. L.; Wei, Y. M., Metabonomic analysis of the anti-inflammatory effects of volatile oils of Angelica sinensis on rat model of acute inflammation. Biomed Chromatogr 2015, 29, (6), 902-10.

36. Abu Bakar, M. H.; Azmi, M. N.; Shariff, K. A.; Tan, J. S., Withaferin A Protects Against High-Fat DietInduced Obesity Via Attenuation of Oxidative Stress, Inflammation, and Insulin Resistance. Appl Biochem Biotechnol 2019, 188, (1), 241-259.

\section{Figures}



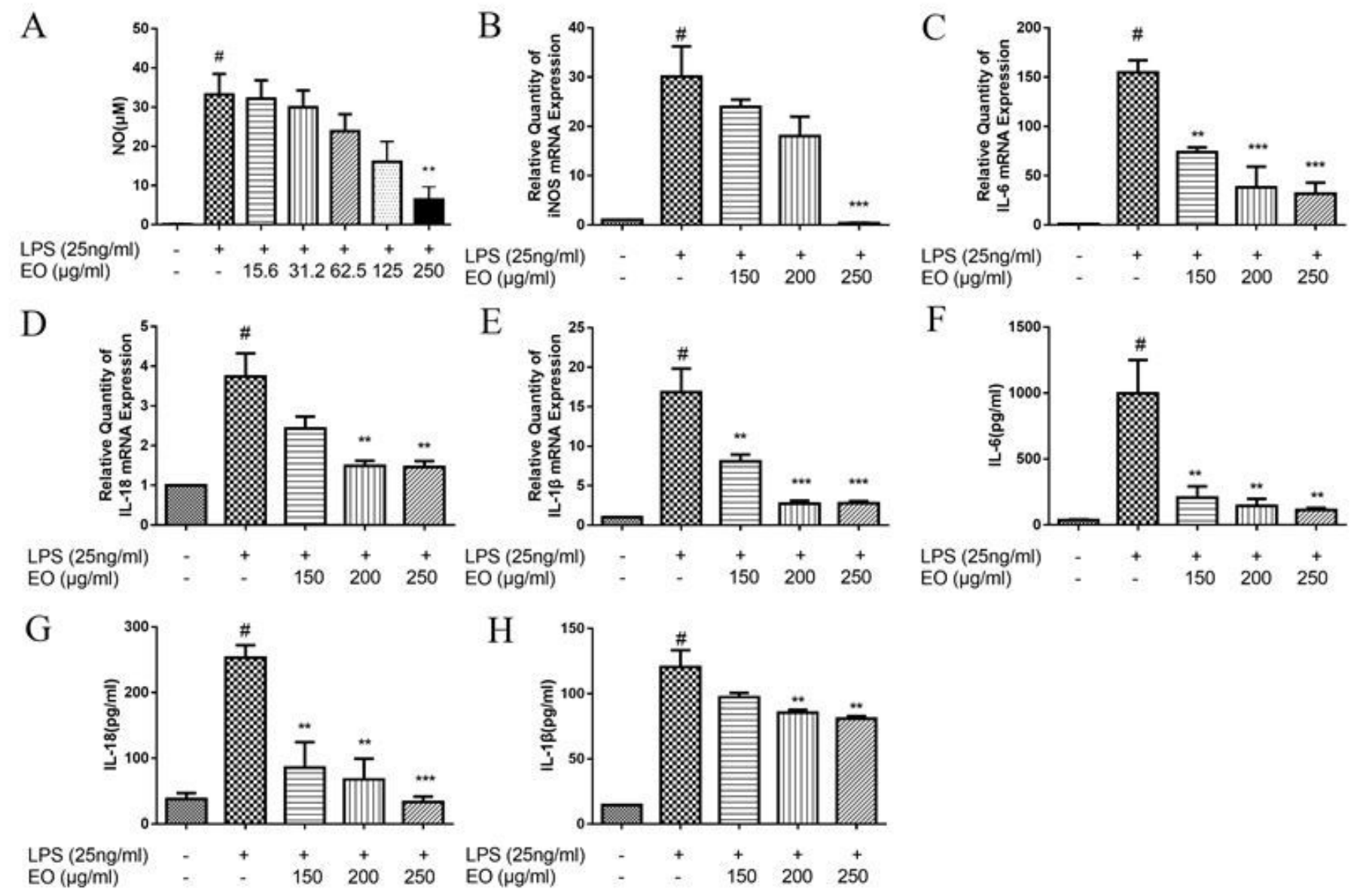

\section{Figure 1}

Effects of EO on LPS-induced production of NO (A) and mRNA expression of iNOS (B). Effects of EO on LPS-induced mRNA expression levels of IL-6 (C), IL-18 (D) and IL-1 $\beta$ (E) in BV2 microglial cells, and secretion of IL-6 (F), IL-18 (G) and IL-1 $\beta(H)$ in the culture supernatant from BV2 microglial cells. $(\# P<$ 0.01 vs. control, $* * P<0.01,{ }^{\star * \star} P<0.001$ vs. LPS treatment, $n=3$ ) 

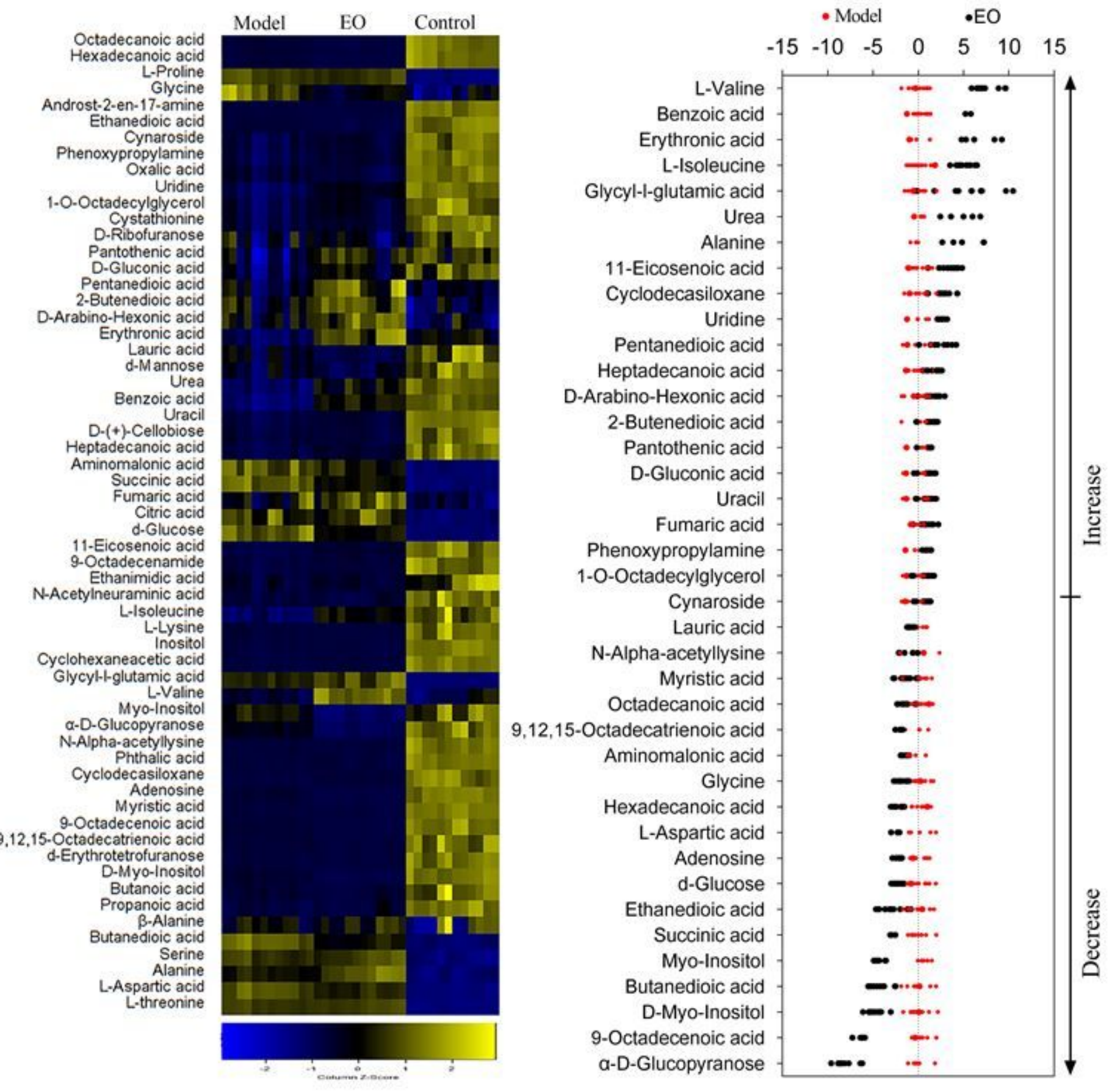

Figure 2

Metabolic profiles of BV2 microglial cells in response to EO. (A) Heat maps of metabolites (row). Yellow and blue indicate an increase and decrease of the metabolites relative to the mean and standard deviation of the row metabolite level, respectively (see color scale). (B) Z-score scatter diagrams of significant differentially expressed metabolites $(P<0.05)$ in the presence or absence of EO. Metabolites are showed on the y-axis. 

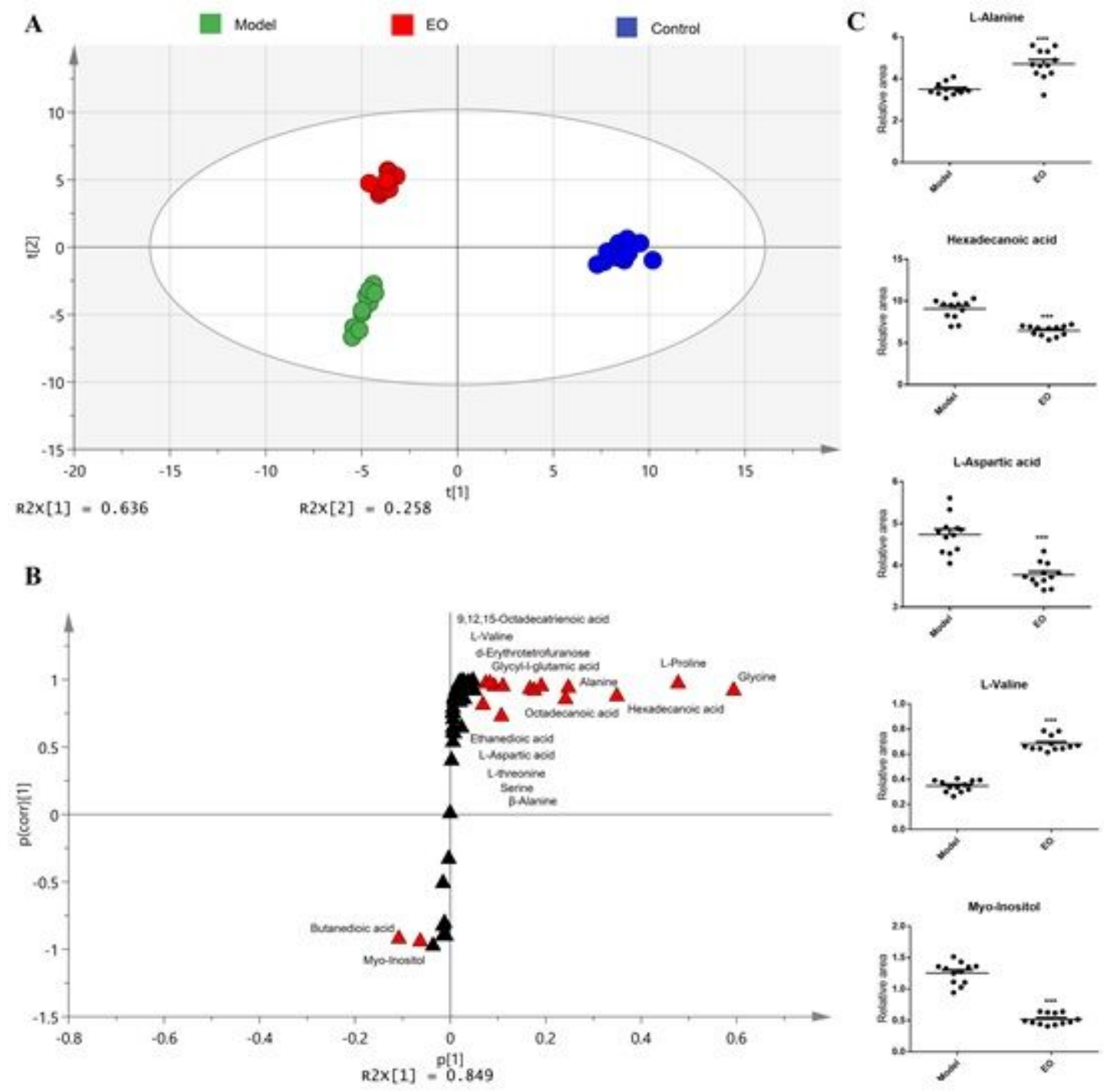

Figure 3

(A) PCA analysis of three groups. Each dot represents the technological replicate analysis of samples. (B) S-plot generated from OPLS-DA. Dot represents metabolites, which are $p[1]<-0.05$ or $>0.05$ and $p$ (corr) [1] $<-0.5$ or $>0.5$ and marked in red dot. (C) Scatter diagram of 5 biomarkers, each dot shows a technical replicate. $n=6$. ${ }^{\star * *} P<0.001$ vs model group. 
A

Impact

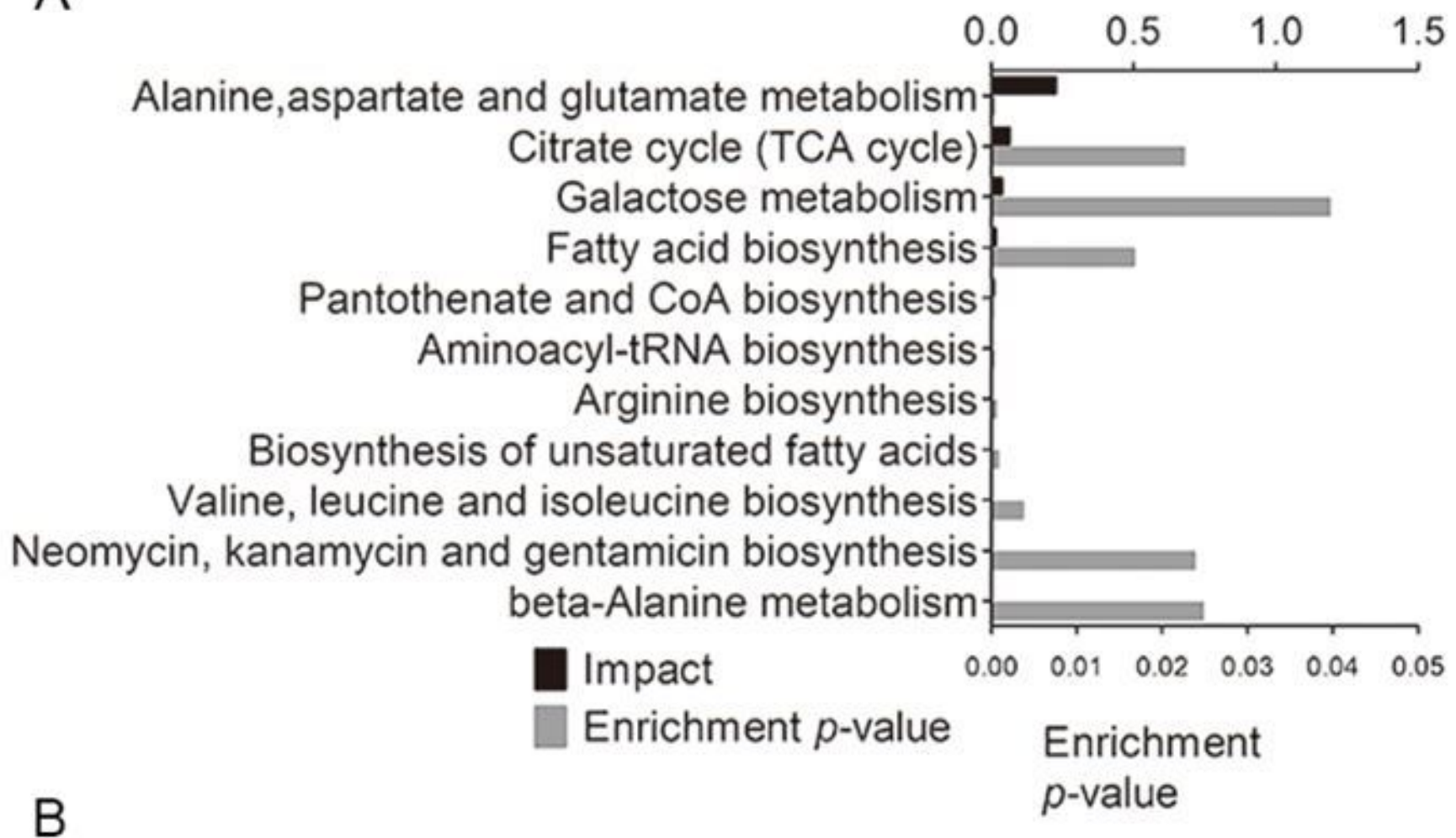

Alanine, aspartate and glutamate metabolism

\begin{tabular}{lr|r|} 
L-Aspartate & 4.7347 & 3.7718 \\
\hline L-Alanine & 3.4994 & 4.7073 \\
Fumarate & -0.1060 & -0.0623 \\
\hline Succinate & 0.0764 & -0.0567 \\
\hline
\end{tabular}

Fatty acid biosynthesis
Hexadecanoic acid

Tetradecanoic acid

Dodecanoic acid

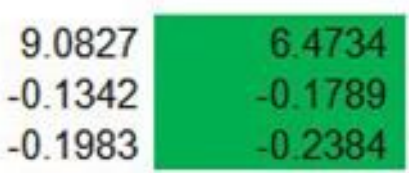

Galactose metabolism

D-Glucose

myo-Inosito
0.2686

1.2549
Citrate cycle (TCA cycle)

Succinate

0.0764

Fumarate

$-0.1060$

Pantothenate and $\mathrm{CoA}$ biosynthesis

Pantothenate

$-0.3189$

L-Valine

0.3471

L-Aspartate

Uracil

4.7347

$-0.3064$
$-0.0567$

$-0.0623$

\section{Figure 4}

(A) Enriched pathways in the presence of the EO significantly enriched pathways were selected to plot. (B) Heat map of the average levels of differential metabolites in the five enriched pathways shared by the two group. Red and green, respectively, indicate increase and decrease in the metabolites scaled to mean and standard deviation of row metabolite level (see the color scale). 

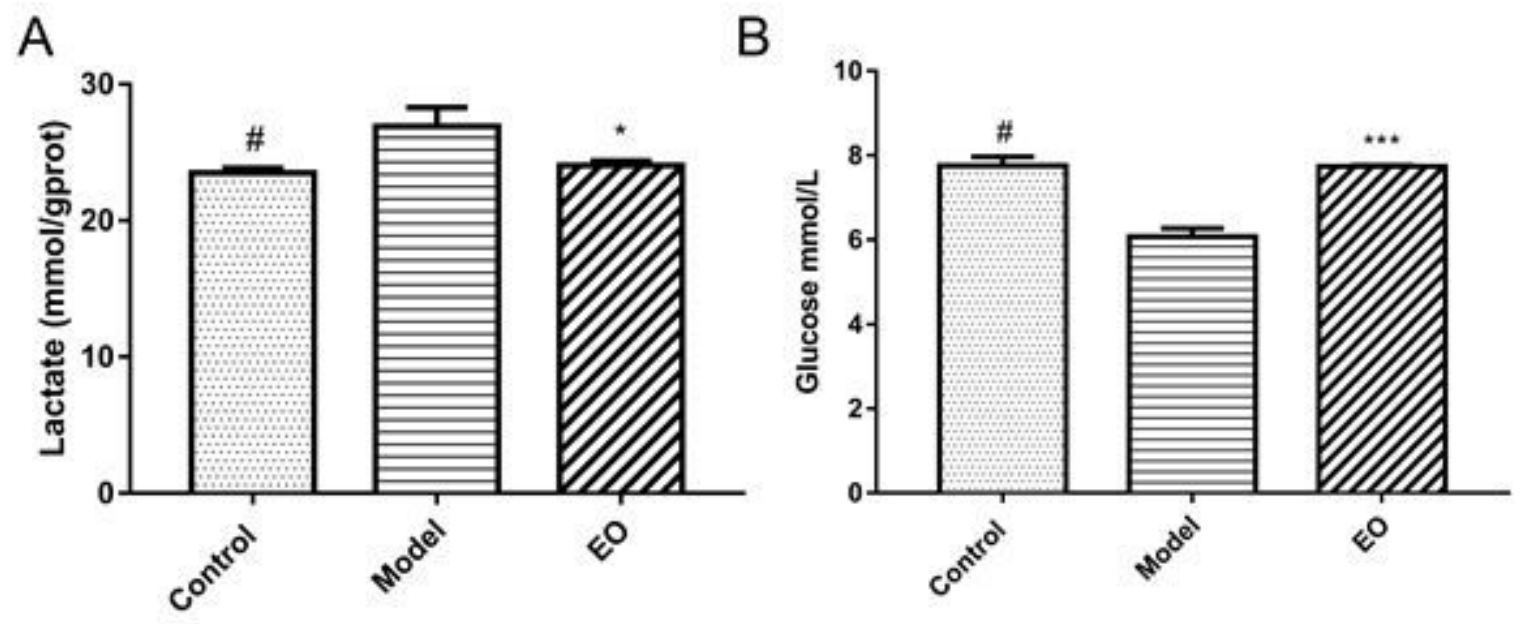

Figure 5

Measurement of the lactate $(A)$ and glucose $(B)$ levels in the supernatant of BV2 microglial cells. \#P< 0.01 vs. control; $* \mathrm{P}<0.05 ; * \star \star \mathrm{P}<0.001$ vs model group. All tests were performed in triplicate.

A

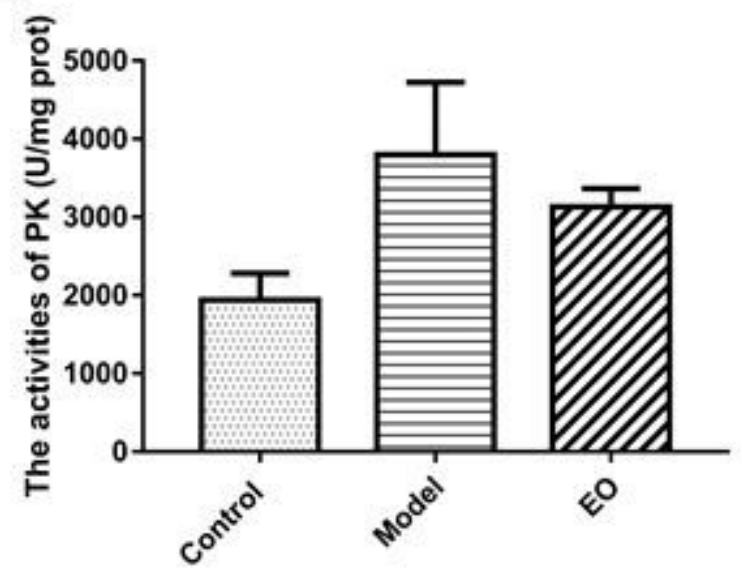

$\mathrm{B}$

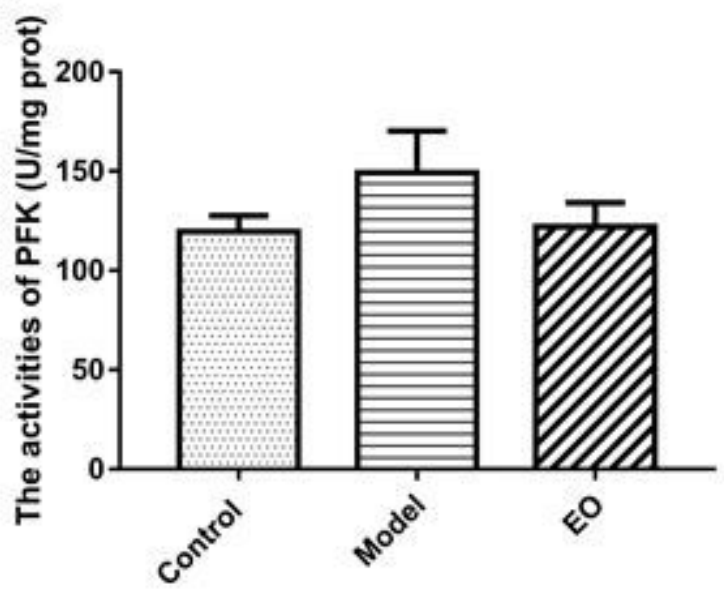

Figure 6

Measurement of the EO on PK (A) and PFK (B) activities in BV2 microglial cells. All tests were performed in triplicate. All tests were performed in triplicate. 